\title{
Safety evaluation of intratympanic and intravenous dexamethasone for sudden sensorineural hearing loss and differences in hearing
}

\section{recovery}

\author{
Jie Huang ${ }^{1}$, Li Yang ${ }^{2}$, Xihong $\mathrm{Cao}^{3}$, and wuqing wang ${ }^{4}$ \\ ${ }^{1}$ The affiliated BenQ Hospital of Nanjing Medical University \\ ${ }^{2}$ The People's Hospital of Xinjiang Uygur Autonomous Region \\ ${ }^{3}$ Mianyang Science City Hospital \\ ${ }^{4}$ Eye Ear Nose \&Throat Hospital, Fudan University
}

July 13, 2020

\begin{abstract}
Objectives: To evaluate the safety of intratympanic and intravenous dexamethasone administration for the treatment of sudden sensorineural hearing loss(SSNHL) and hearing improvement at low, mid and high frequencies. Methods: SSNHL patients were randomly divided into two groups within 72 hours after onset and received 24 days of dexamethasone therapy.The group A patients only received intravenous dexamethasone once every other day.Patients in group B received 12 days intravenous dexamethasome and six injection of intratympanic dexamethasome from the 13th day to 24th day like in Group A.Side effects and hearing recovery were compared. Results: The discomfort of intratympanic injection were mild and transient, no tympanic membrane perforation and otitis media, whereas the systemic adverse effects increased cumulatively over the course of intratympanic dexamethasone treatment in group A.The systemic side effects were more serious in group B than in group A. On day 7 (D7), the average 3-frequency PTA (250, 500, and $1000 \mathrm{~Hz})$ was 34.10 $\pm 9.14 \mathrm{dBHL}$ in group A and 25.70 $\pm 6.88 \mathrm{dBHL}$ in group $\mathrm{B}(\mathrm{P}<0.05)$. Hearing recovery was better in group $\mathrm{A}$ than group $\mathrm{B}$, but there are the same long-term efficacy at the 90-day follow-up.Group A had slightly better high-frequency hearing recovery than group B, but this difference was not statistically significant. There were no significant differences in the total effective rate of hearing improvement. Conclusions: Early intratympanic dexamethasone intervention led to the rapid recovery of hearing loss, with a good remedial effect on hearing at low-mid frequencies. Intratympanic dexamethasone was safer than intravenous dexamethasone for SSNHL, and high-frequency hearing recovery may have been better with intratympanic dexamethasone, but the difference was not statistically significant. Therefore, it is necessary to make individualized treatment decisions according to the patient's condition.
\end{abstract}

\section{Introduction}

Sudden sensorineural hearing loss (SSNHL) affects approximately 5 to 27 per 100,000 people annually in the United States, similar to the case frequency in other countries, and this incidence has been increasing gradually ${ }^{[1-3]}$. Corticosteroids alone or combined with hyperbaric oxygen therapy have been shown to be effective and are widely utilized by otologists. However, the range of therapeutic strategies remains various because of the uncertain comparative efficacy and safety of competing treatments. A recent systematic review and network meta-analysis of existing pharmaceuticals included a large number of studies involving patients who received intratympanic, intravenous plus+intratympanic, and oral steroids treatment. Although the results were unclear due to the high risk of bias, they rated intratympanic plus systemic steroid treatment as the best of the six included interventions ${ }^{[1]}$. However, in other studies, intratympanic steroids (ITSs) led to better outcomes, including PTA improvement and a better recovery rate, than intravenous steroid treatment ${ }^{[2]}$. This study evaluated the efficacy and side effects of initial ITSs and intravenous plus intratympanic ste- 
roids, which were administered over a longer course than that used incommon practice for patients diagnosed with SSNHL.

\section{Patients and methods}

\section{Patients}

From January 2013 to October 2018,patients were admitted after being diagnosed with SSNHL and randomly divided into two groups. Inclusion criteria: 1. Initial onset of unilateral SSNHL within 72 hours; 2. Hearing loss of $30 \mathrm{~dB}$ or greater for three consecutive frequencies by PTA $(0.25,0.5,1,2,4$, and $8 \mathrm{kHz}) ; 3$. At least 18 years old but younger than 65 years; and 4. Patient consent. Exclusion criteria: 1. Unmanaged serious systemic diseases, diabetes or hypertension; epilepsy; psychosis; active ulcer of the digestive tract; or any contraindications for hormone therapy; 2. Identifiable aetiology for hearing loss, such as radiation, noise, acute or chronic otitis media, Meniere's disease, autoimmune diseases, large vestibular aqueduct syndrome, and retrocochlear disease.

\section{Injection technique}

The intratympanic injection procedure was performed in an endoscopic ear surgery room. Topical anaesthesia with $1.5 \%$ tetracaine was administered for 20 minutes. Patients were placed in a supine position, with their head tilted 40 to 45 degrees toward the side of the healthy ear, and the solution was then perfused into the middle ear after myringotomy. Following injection, the patients were advised to avoid moving their head, speaking or swallowing for 30 minutes.

\section{Treatment protocol}

The patients were randomized to groups A(intratympanic dexamethasone) and B (intratympanic plus intravenous dexamethasone). Patients in both groups whose hearing did not recover received the following treatments for 24 days. In group A, dexamethasone was given once every other day for 24 days $(5 \mathrm{mg} / \mathrm{ml}$, $1.5 \mathrm{mg} / 0.3 \mathrm{ml})$. In group B, intravenous dexamethasone was administered for 12 days $(10 \mathrm{mg} / \mathrm{d}$ on days 1-4, $7.5 \mathrm{mg} / \mathrm{d}$ on days $5-8$, and $5 \mathrm{mg} / \mathrm{d}$ on days 9-12), and intratympanic dexamethasone was then given once every other day for the following 12 days.

\section{Efficacy evaluation}

\section{Audiometric data}

Six-frequency PTA was performed before the start of treatment (D1) and on days 7 (early test point, D7), 13 (middle test point, D13), 24 (late test point, D24), and 90 (long-term test point, D90) or until the patient fully recovered. The mean PTA values at low-mid frequencies $(0.25,0.5$, and $1 \mathrm{kHz})$ and high frequencies $(2$, 4 , and $8 \mathrm{kHz}$ ) were calculated. The total effective rate was the sum of complete, marked and slight hearing gain (PTA $>15$ dBHL) according to Siegel's criteria ${ }^{[3]}$.

\section{Side effects}

Local and systemic side effects of steroid therapy were observed, such as vertigo, tympanic membrane perforation, middle ear infection, fluctuations in basal blood pressure (BBP) and fasting blood glucose (FBG) and other systemic adverse effects.

\section{Statistical analysis}

The results are presented as the mean (SD). Two-sample $t$ tests and $\chi^{2}$ tests were used to compare the effects in the two groups. The $\mathrm{P}$ value of 0.05 was considered to indicate statistical significance. All analyses were performed using SPSS software 19.0.

\section{Results}

A total of 227 newly diagnosed SSNHL patients were admitted.104 patients fulfilled the inclusion criteria, provided informed consent, and were enrolled in the two groups. Five patients, 3 in group A and 2 in group 
B, refused the IT injections due to unbearable pain. Steroid treatment was stopped for one patient in group B because of difficulty controlling blood glucose levels under control. There were no significant differences between groups in age, sex, classification of audiogram, or complications such as diabetes and hypertension (Table 1).

\section{Side effects}

\section{Systemic side effects}

Ten systemic side effects of steroids were observed. More indications of systemic side effects were observed in group $\mathrm{B}(\mathrm{n}=139)$ than in group $\mathrm{A}(\mathrm{n}=67)$ (Table 2).

In group $\mathrm{A}$, the systemic side effects were no more serious than slight changes in appetite or emotions or difficulty falling asleep during the initial treatment stage in week one. Symptoms such as water and sodium retention, facial acne, and fluctuations in blood pressure and glucose levels appeared as the treatment continued, specifically after 8 doses of intratympanic dexamethasone, and gradually recovered after drug withdrawal. During the course of intravenous plus intratympanic dexamethasone therapy in group B, systemic side effects increased in response to cumulative drug exposure. Dyssomnia, changes in appetite, edema caused by water and sodium retention, emotional changes and fluctuations in FBG were the most common complications. These patients showed obvious changes in appetite and emotions, facial congestion, nocturnal sleep disorders and fluctuations in BBP during the early stage of treatment. In one patient, steroid therapy was stopped because of uncontrolled glucose levels.

There were no differences between the two groups in the incidence of emotional changes, acne, menstrual disorders, or fluctuations in BBP $(>10 \mathrm{mmHg})$ or FBG. There were difference between groups was observed in appetite changes, and there were significant differences were observed in dyssomnia and oedema caused by water and sodium retention. A mild Cushing's state was detected 1 month after therapy in one patient in group B. No brittle fractures occurred in any patient.

\section{Local side effects}

Three patients in group A and two patients in group B refused repeated intratympanic injection due to unbearable pain. Twenty patients in the two groups complained of obvious transient vertigo after IT injection, but none of these patients terminated treatment. There were no cases of apparent bleeding, external otitis, otitis media or perforation of the tympanic membrane.

\section{Recovery of overall hearing}

The total hearing improvement at the long-term test point (D90) after admission was 31.0 9 9.64 dBHL in group A and 29.4 $\pm 9.18 \mathrm{dBHL}$ in group B. An effective rate of hearing improvement [?]15 dB was noted in 43 of the 49 patients in group A (87.8\%) and 42 of the 49 patients in group B (85.7\%). There were no significant differences between the two groups(Table 3 ).

\section{Recovery of hearing loss atlow-mid frequencies}

On day 7 (D7), the average 3 -frequency PTA (250, 500, and $1000 \mathrm{~Hz})$ value was $34.10+-9.14 \mathrm{dBHL}$ in group A and 25.70+-6.88 dBHL in group B. Hearing recovery was better in group A than group B, and statistical comparison of the two groups by a t test $(\mathrm{P}<0.05)$ showed a significant difference between intratympanic dexamethasone(group A) and intravenous dexamethasone(group B). On days 13, 24 and 90 (D13, D24, D90), the average PTA values were 39.55+-7.03, 42.15+-6.65, and 42.70+-6.57 dBHL in group A and 35.05+-6.35, $41.85+-5.38$, and $42.20+-5.12 \mathrm{dBHL}$ in group B, respectively; there were no significant differences between group $\mathrm{A}$ and group $\mathrm{B}(\mathrm{P}>0.05)$. The results showed rapid hearing recovery at low and mid frequencies in group A, and intratympanic plus intravenous dexamethasone in group B showed the same long-term efficacy with group A at the 90-day follow-up(Figure 1).

\section{Recovery of hearing loss athigh frequencies}


On day 7 (D7), the hearing improvement for the average 3-frequency PTA (2000, 4000, and $8000 \mathrm{~Hz})$ value was 9.75+-3.26 dBHL in group A and 8.85+-2.93 dBHL in group B $(\mathrm{P}>0.05)$; thus, there was no significant difference between groups in hearing recovery at high frequencies. On D13, D24 and D90, the hearing improvements at average high frequencies were 18.90+-5.72, 20.95+-5.50, and 21.75+-5.95 dBHL in group A and 17.35+-4.87, 19.80+-6.18, and 20.55+-6.19 dBHL in group B. Group A exhibited better hearing improvement $(21.75+-5.95 \mathrm{dBHL})$ than in group B $(20.55+-6.19 \mathrm{dBHL})$, but the difference between groups was not significant $(\mathrm{P}<0.05)$. intratympanic dexamethasonemay lead to better hearing recovery at high frequencies than intravenous dexamethasone, but the difference was not significant in this study (Figure 2).

\section{Discussion}

The possible causes of SSNHL include virus infection, autoimmune reactions, and vascular insults ${ }^{[4-6]}$, which evoke similar pathophysiological processes, such as cochlear ischemia and hypoxia. In this study, the results were evaluated by PTA and some adverse effects. Steroids are critical for the treatment of SSNHL because of their anti-inflammatory activity, ability to eliminate cochlear oedema and immunosuppressive activity. However, it is difficult to ascertain the safety and efficacy of these heterogeneous steroid therapies based on results from biased studies ${ }^{[7-9]}$.

Many treatments for SSNHL, including systemic or local steroid application ${ }^{[10]}$ and HOT, are effective. In 1980, Wilson et al. ${ }^{[16]}$ demonstrated in a double-blind controlled study that the hearing recovery rate was $78 \%$ in the steroid group, which was significantly better than the rate of $38 \%$ observed in the placebo group. Silverstein et al. ${ }^{[17]}$ first reported that the tympanic injection of glucocorticoids is effective for SSNHL. Since that time, ITSs have become popular and have shown equivalent efficacy to systemic steroids. Arnold et $\mathrm{al}^{[11]}$ reported that tympanic injection did not cause structural injury in the round window of the cochlea or in inner and outer cochlear hair cells. Obstructions in the round window niche and abnormal permeability of the round window membrane that prevent drugs from being effective are rare. In animal models ${ }^{[12,13,14]}$, ITSs administration resulted in significantly greater drug concentrations in the perilymph than did compared with systemic administration. Moreover, glucocorticoid levels were even higher in the cochlear apex, and a decreasing basal-apical concentration gradient along the scala tympani was detected after round window application. Amandeep S Grewal et al. reported similar drug concentrations in the cochlea after IV and intratympanic administration, although the intravenous dexamethasone concentrations used were higher than those applied in clinical practice ${ }^{[15]}$. It is not sufficient to only understand steroid pharmacokinetics in the inner ear; the therapeutic outcome also depends on the distribution and regulation of glucocorticoid receptors in the cochlea. Many studies have shown that ITSs are effective and relatively safe ${ }^{[18]}$, and all of the side effects are either transient or easily curable. However, ITSs enter the digestive tract through the eustachian tube and are inevitably absorbed and accumulated in the body. The safety of drug application routes should be closely monitored by otologists as there are no standard responses.

The role of steroids in the treatment of SSNHL has been confirmed in many randomized double-blind trials. Some studies showed that intratympanic steroid injection leads to better hearing outcomes, while other studies have not reached this conclusion. There are obvious differences in the studies of steroid treatment for SSNHL, such as grouping errors, the use of different steroids, differing frequencies of administration, differences in the timing of treatment initiation and treatment duration, and the administration of mixed lidocaine; all these factors can lead to heterogeneity in the results. The natural recovery and good prognosis of low-mid frequency hearing (large air conduction gap before and after steroid treatment) are statistically dominant over those of high frequency hearing (narrow air conduction gap), which may explain the inconsistent research conclusions. Multiple SSNHL guidelines refer to intratympanic dexamethasoneas salvage therapy. For instance, The Otolaryngology Association of Madrid's consensus statement recommends intratympanic corticosteroids if a complete response to systemic steroids does not occur after 7 days ${ }^{[22]}$. Clinicians may offer corticosteroids with HOT as initial therapy to SSNHL patients within 3 months of diagnosis per the American Academy of Otolaryngology-Head and Neck Surgery's clinical practice guideline ${ }^{[23]}$. In 2019, clinicians were recommended to offer ITS when patients showed an incomplete recovery from SSNHL 2 to 6 weeks after the onset of symptoms (KAS 10). In Germany and Austria, $49.1 \%$ of otolaryngologists prescribe 
ITSs for ISSNHL, but of these otolaryngologists, $73.7 \%$ do not prescribe it as primary treatment. A total of $20.6 \%$ of otolaryngologists administer ITSs in conjunction with oral steroids for primary treatment, only $5.8 \%$ administer ITSs as monotherapy for primary treatment, and $90.5 \%$ utilize ITSs as salvage therapy; $81.1 \%$ did not consider the use of ITS for 2 weeks after the onset of symptoms ${ }^{[24]}$.In practice, among patients who showed no hearing improvement on prior intravenous steroid treatment, some experience significant hearing improvement after intratympanic treatment. Therefore, is it a paradox that the two therapies are believed to be equally effective? Should ITS be recommended after intravenous steroid treatment because of the mild systemic side effects? Can the mild long-term side effects of steroids be ignored?

The most commonly used steroids for SSNHL treatment are dexamethasoneand methylprednisolone, but different drug concentrations, dosages, densities, and durations are implemented. Parnes and Shirwany et al. ${ }^{[19,20]}$ showed that the permeability of dexamethasonein round window membranes is lower than that of methylprednisolone. Trune and Kempton ${ }^{[21]}$ found that methylprednisolone has a 1.7 -fold stronger affinity for hormone receptors in the inner ear than dexamethasone. However, other researchers have argued that the higher concentrations of methylprednisolone in the endolymph were due to decreased absorption by cochlear and vestibular tissues. Earache after methylprednisolone intratympanic injection led to more patient intolerance significantly than dexamethasone.No high-quality research study favours one conclusion over the other.

Dexamethasone is most commonly administered at $4 \mathrm{or} 5 \mathrm{mg} / \mathrm{ml}(0.2-25 \mathrm{mg} / \mathrm{ml} ; 4 \mathrm{mg} / \mathrm{ml}$, approximately $60 \%)$ and is readily absorbed in the digestive tract. The half-life $\left(\mathrm{T}_{1 / 2}\right)$ of dexamethasone is 190 minutes in plasma and 3 days in tissue.In this study, we administered dexamethasone at a concentration of 5 $\mathrm{mg} / \mathrm{ml}$. Rapid hearing improvement was observed at low-mid frequencies in group A (D7), suggesting that intratympanic dexamethasone promoted earlier hearing recovery than intravenous dexamethasone. However, the long-term results were equivalent in group A and group B, which received intratympanic dexamethasone after 12 days of intravenous plus intratympanic dexamethasone treatment. These results indicate that intratympanic dexamethasone has a good salvage effect, it not means the equivalent effects between intratympanic and intravenous treatment. The hearing improvement at high frequencies was slightly better in group A than in group B, but the difference between groups was not significant. ITSs promote early and better hearing recovery due to rapid tissue distribution in the inner ear and the high concentration of dexamethasone near the tympanic medial wall.Different degrees of hearing recovery occurred at different frequencies, and the previous contradictory research results may be caused by audiological characteristics. It is difficult to define the curative overall hearing effect because of the good hearing prognosis at low frequencies and the modest difference in hearing improvement at high frequencies.

Furthermore, drug safety limits the use of steroids. After obtaining informed consent from patients, we developed a 24-day steroid treatment plan for patients who did not have a complete recovery. The slight local adverse effects included mild pain at the injection site and short-term vertigo, and no persistent tympanic membrane perforations occurred. Obvious systemic side effects were observed, such as changes in appetite, limb oedema, dyssomnia, menstrual disorders, and fluctuations in blood pressure and glucose levels, which were more serious in group B than in group A. One patient developed Cushing's syndrome 2 months after steroid therapy; this outcome has not been reported previously for short-term treatment protocols. During the course of intratympanic dexamethasone treatment in group A, systemic adverse reactions were accompanied by the inevitable accumulation of dexamethasone and increased accordingly, but there were still fewer reactions than with intravenous plus intratympanic combination therapy. Therefore, intratympanic injection of corticosteroids is superior to intravenous steroids for hearing improvement after SSNHL and is safer, especially for patients with systemic diseases. intravenous steroid administration deserves serious consideration only in the following situation: SSNHL patients with other autoimmune damage or damage beyond the range of the membranous labyrinthine. In addition, a good self-healing effect was observed in patients only with low-mid frequency hearing impairment, the invasive intratympanic injection should be applied conservatively.

Conclusions: Intratympanic dexamethasone promotes rapid hearing recovery and has a good salvage effect 
at low-mid frequencies. It potentially exhibits better performance in high-frequency hearing recovery, but the difference was not statistically significant. In this study, the intratympanic dexamethasone is more safer than intravenous steroids.It is necessary to make individualized treatment decisions according to patient age, the presence of vertigo, hearing loss characteristics of PTA, underlying systemic diseases, and tolerance to repeated intratympanic injections.

\section{List of abbreviations}

SSNHL:Sudden sensorineural hearing loss.

PTA:Pure-tone threshold audiometry.

ITSs:Intratympanic steroids.

BBP:Basal blood pressure.

FBG:Fasting blood glucose.

\section{Declarations}

No conflict of interest exits in the submission of this manuscript, and manuscript is approved by all authors for publication. I would like to declare on behalf of my co-authors that the work described was original research that has not been published previously, and not under consideration for publication elsewhere, in whole or in part. All the authors listed have approved the manuscript that is enclosed.

\section{References}

[1] Ahmadzai N, Kilty S, Cheng W, Esmaeilisaraji L, Wolfe D, Bonaparte JP, et al. (2019) A systematic review and network metaanalysis of existing pharmacologic therapies in patients with idiopathic sudden sensorineural hearing loss. PLoS ONE 14(9): e0221713.

[2]Qiang Qingfen,Wu Xuewen,Yang Tao,Yang Chunguang,Sun Hong. A comparison between systemic and intratympanic steroid therapies as initial therapy for idiopathic sudden sensorineural hearing loss: a metaanalysis. Acta oto-laryngologica.2017,137(6):598-605.

[3] L G, Siegel.The treatment of idiopathic sudden sensorineural hearing loss.Otolaryngologic clinics of North America.1975,8(2):467-73.

[4] Yamasoba T, Kikuchi S, Sugasawa M, et al. Acute low-tone sensorineural hearing loss without vertigo. Arch Otolaryngol Head Neck Surg.1994,120(5) : 532-535.

[5] Fuse T,Hayashi T,Oota N. Immunological responses in acute low-tone sensorineural hearing loss and Meniere's disease[J].Acta Oto-Laryngologica,2003,(01):26-31.

[6]Takahashi M,Odagiri K,Sato R,et al. Personal factors involved in onset or progression of Meniere's disease and low-tone sensorineural hearing loss.Oto-rhino-laryngology.2005,67(5):300-04.

[7] Edizer D T,Celebi O,Hamit B,et al. Recovery of Idiopathic Sudden Sensorineural Hearing Loss. Journal of International Advanced Otology.2015,11(2):122-26.

[8]Chung J H,Cho S H,Jeong J H,et al.Multivariate analysis of prognostic factors for idiopathic sudden sensorineural hearing loss in children.The Laryngoscope.2015,125:2209-15.

[9]Suzuki H,Wakasugi T,Kitamura T,et al.Comparison of 2 and 4 Intratympanic Steroid Injections in the Treatment of Idiopathic Sudden Sensorineural Hearing Loss. Annals of Otology, Rhinology \& Laryngology.2018,127:235-40.

[10] Sutton L,Schartinger V,Url C,et al.Intratympanic steroid use for idiopathic sudden sensorineural hearing loss: current otolaryngology practice in Germany and Austria.European Archives of Oto-RhinoLaryngology.2018,275:1103-10. 
[11] Arnold W,Senn P, Hennig M , et al. Novel Slow- and Fast-Type Drug Release Round-Window Microimplants for Local Drug Application to the Cochlea: An Experimental Study in Guinea Pigs.Audiology and Neuro-Otology.2005, 10(1):53-63.

[12] Parnes LS,Sun AH, Freeman DJ. Corticosteroid pharmacokinetics in the inner ear fluids: an animal study followed by clinical application. Laryngoscope.1999;109:1Y17.

[13]Grewal AS,Nedzelski JM,Chen JM,et al.Dexamethasone uptake in the murine organ of Corti with transtympanic versus systemic administration.J Otolaryngol Head Neck Surg.2013,42:19.

[14]Salt AN: Dexamethasone concentration gradients along scala tympani after application to the round window membrane. Otol Neurotol 2008, 29:401-406.

[15]Amandeep S,Nedzelski Julian M,Chen Joseph M,et al.Dexamethasone uptake in the murine organ of Corti with transtympanic versus systemic administration. Journal of otolaryngology - head \& neck surgery ,2013,42:19.

[16] Wilson WR, Byl FM, Laird N.The efficacy of steroids in the treatment of idiopathic sudden hearing loss. A double-blind clinical study[J]. Arch Otolaryngol, 1980, 106(12) :772-776.

[17] Silverstein H1,Choo D,Rosenberg SI,et al. Intratympanic steroid treatment of inner ear disease and tinnitus (preliminary report).Ear Nose Throat.1996 ,75(8):468-67.

[18]Plontke SK,Lowenheim H,Mertens J,et al. Randomized, double blind, placebo controlled trial on the safety and efficacy of continuous intratympanic dexamethasone delivered via a round window catheter for severe to profound sudden idiopathic sensorineural hearing loss after failure of systemic therapy.Laryngoscope. 2009,119:359-69.

[19] Parnes LS,Sun AH,Freeman DJ.Corticosteroid pharmacokinetics in the inner ear fluids:animal study followed by clinical application. Laryngoscope.1999,109 (7 Pt 2): 1-17.

[20] Shirwany NA, Seidman MD, Tang W.Effect of transtympanic injection of steroids on cochlear blood flow,auditory sensitivity,and histology in the guinea pig. Am J Otol, 1998,19(2): 230-35.

[21] Trune DR, Kempton JB. Aldosterone and prednisolone control of cochlear function in MRL/MpJFas(lpr) autoimmune mice.Hear Res, 2001, 155(1-2): 9-20.

[22] Plaza G, Durio E, Herra'iz C,et al.Consensus on diagnosis and treatment of sudden hearing loss. Asociacio'n Madrilena de ORL]. Acta Otorrinolaringol Esp. 2011 Apr; 62(2):144-57.

[23]Stachler RJ, Chandrasekhar SS, Archer SM, et al. Clinical practice guideline: sudden hearing loss. Otolaryngol-Head Neck Surg Off J Am Acad OtolaryngolHead Neck Surg.2012 Mar; 146(3 Suppl):S1-35.

[24]Matt Lechner,Liam Sutton,Mark Ferguson,et al. Intratympanic Steroid Use for Sudden Sensorineural Hearing Loss: Current Otolaryngology Practice[J]. Annals of Otology, Rhinology \& Laryngology,2019,128(6).

\section{Hosted file}

\begin\{CJK\}\{UTF8\}\{gbsn\}. \end\{CJK\}\selectlanguage\{english\}doc available at https://authorea. } com/users/341549/articles/468426-safety-evaluation-of-intratympanic-and-intravenousdexamethasone-for-sudden-sensorineural-hearing-loss-and-differences-in-hearing-recovery 
Hearing improvement at low-mid frequencies

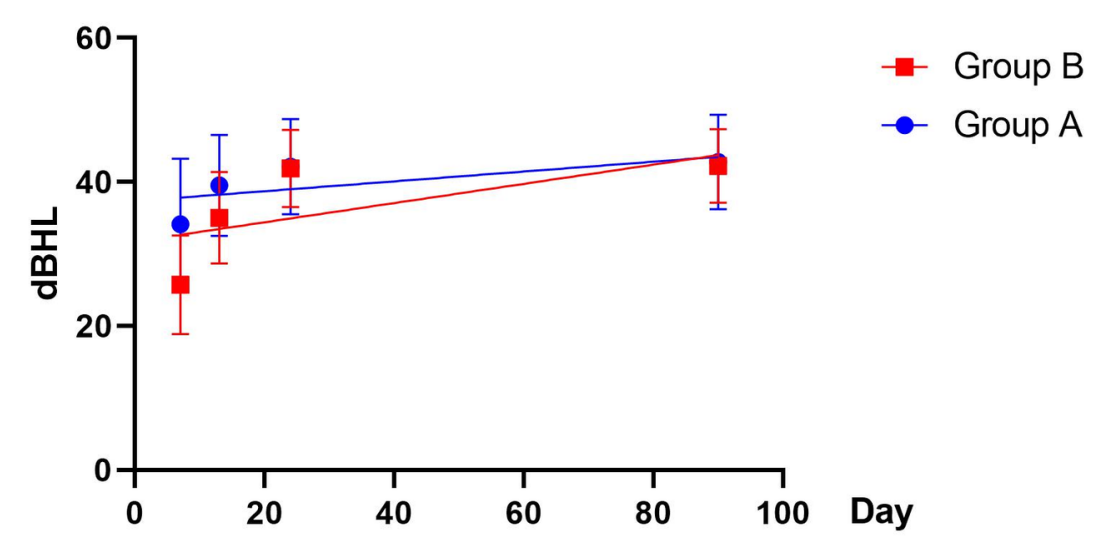

Hearing improvement at high frequencies

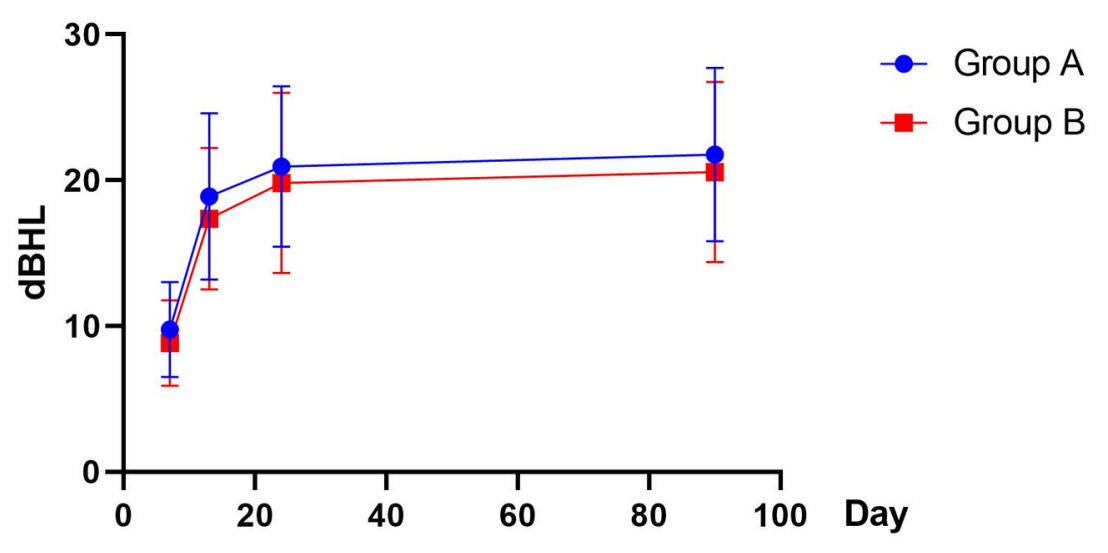

